\section{6 OPEN ACCESS}

\title{
Functional and anatomical outcome of eyes with neovascular age-related macular degeneration treated with intravitreal ranibizumab following an exit strategy regimen
}

\author{
Marcel N Menke, Martin S Zinkernagel, Andreas Ebneter, Sebastian Wolf
}

Department of Ophthalmology, Inselspital, Bern University Hospital, and University of Bern, Bern, Switzerland

\section{Correspondence to} Dr Marcel N Menke, Reinhardweg 6, 3422 Kirchberg, BE, Switzerland marcel.menke@gmail.com

Received 11 December 2013 Revised 4 March 2014 Accepted 20 March 2014 Published Online First 29 April 2014

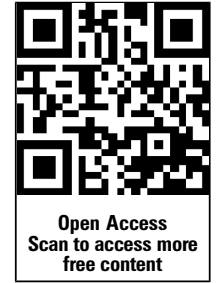

CrossMark

To cite: Menke MN Zinkernagel MS, Ebneter A, et al. Br J Ophthalmol 2014;98:1197-1200.

\section{ABSTRACT}

Aims To assess the functional and morphological outcome of eyes with neovascular AMD treated with intravitreal ranbizumab following an exit strategy treatment regime.

Methods The Bern treatment regime for neovascular AMD has a fixed injection schedule, even in the nonactive stage of the disease. The regimen has been adapted from the PIER study treatment protocol. Eyes with non-active AMD will receive 4 injections in the first year, and 2 injections in the second year of follow-up before treatment stops. Patients that received ranibizumab for treatment and reached the exit criteria were identified, and charts were reviewed to assess functional and morphological outcome.

Results Only $2.6 \%$ of all patients (15 out of 575 patients) reached the exit criteria. Mean change in best corrected ETDRS visual acuity (VA) was $4.5 \pm 16.9$ letters when comparing baseline VA to 4 weeks after the last injection ( $p=0.32$ ). OCT mean foveal thickness was significantly thinner after last treatment (247.9 $\pm 43.0 \mu \mathrm{m})$ compared to baseline $(332.5 \pm 83.1 \mu \mathrm{m}$, $p=0.002$ ). The mean total number of ranibizumab injections was $15.6 \pm 8.0$, and the mean total treatment period was $40.9 \pm 18.3$ months. Twenty percent of eyes had geographic atrophy present at baseline versus $46.6 \%$ at the end of treatment.

Conclusions Even with a fixed treatment regime and a defined treatment exit strategy, only a small percentage of patients reach exit criteria. Retinal thickness has been significantly reduced by repeated intravitreal ranibizumab injections, and geographic atrophy became more frequent.

\section{INTRODUCTION}

Age-related macular degeneration (AMD) is the leading cause of visual impairment and irreversible blindness among elderly people over 50 years of age in the Western world. ${ }^{1}$ Vascular endothelial growth factor-A (VEGF-A) has been identified as the key factor for the pathogenesis of exudative AMD, which has the worst prognosis. ${ }^{2}$ Over the last 10 years, a number of new antibodies against VEGF-A emerged for intraocular treatment. Ranibizumab is a recombinant, humanised, monoclonal antibody Fab fragment that inhibits all biologically active VEGF-A isoforms. ${ }^{3}$ It is indicated in active neovascular AMD with evidence of intraretinal or subretinal fluid by optical coherence tomography (OCT), intraretinal and subretinal haemorrhage, enlargement of choroidal neovascularisation (CNV) size on fluorescence angiography, and when there is a new or persistent leakage on fluorescence angiography. ${ }^{4}$

The International Retina Expert panel has recommended an evidence-based guideline to optimise treatment outcome with ranibizumab in neovascular AMD. ${ }^{6}$ This recommendation is based on the evidence available from prospective, multicentre studies evaluating different ranibizumab treatment schedules, such as ANCHOR, ${ }^{7}$ MARINA, ${ }^{8}$ PIER, ${ }^{9}$ SAILOR, ${ }^{10}$ SUSTAIN $^{11}$ and EXCITE. ${ }^{12}$ In clinical routine, many different treatment regimes for ranibizumab are available (for example: monthly injections, PRN, treat and extend). Although criteria are relatively clear regarding when to start antiVEGF treatment, there is quite some uncertainty when to stop therapy.

In the Bern treatment regime, patients receive three monthly injections during the loading phase. After the loading phase, patients were initially followed monthly and treated pro re nata. Patients were closely followed with OCT, ETDRS visual acuity testing and fundus biomicroscopy during each follow-up, and in case of relapse with disease activity, the regime would start with a reloading phase over again. ${ }^{13}$ Every retreatment due to new disease activity triggered three additional monthly injections. In case of no disease activity, patients received fixed injections every 3 months for a period of 1 year. In the second year without disease activity, two more injections were performed after 6 months and 12 months, after the last injection therapy was stopped. Figure 1 shows a scheme of the Bern treatment regime. The purpose of this study was to identify patients who finished treatment following the complete Bern treatment regime to assess functional and anatomical outcome.

\section{PATIENTS AND METHODS}

All patients treated with intravitreal antiVEGF drugs at the Department of Ophthalmology, Inselspital, University Hospital Bern, Switzerland, are registered in an institutional database. Local IRB-approved retrospective analysis of data for this study. Charts of patients who received intravitreal injections with ranibizumab for neovascular AMD were identified. Treatment had to be started after 12/2008 to assure that the Bern treatment regime was applied. All treatments were performed between 12/2008 and 3/ 2013. No previous treatment for neovascular AMD was allowed in this study. Out of 1295 identified patients who received ranibizumab for neovascular AMD, 335 patients had prior treatment with bevacizumab, photodynamic therapy or conventional laser 
Figure 1 Scheme of the Bern treatment regime to treat neovascular AMD with intravitreal ranibizumab injections. Active disease is treated with at least 3 monthly injections (loading phase). Non-active disease is treated with injections every three months during the first year and every six months during the second year. If the disease remains non-active over 24 months, the therapy ends. Any recurrence of disease activity will lead to a new loading phase, and the regime starts over again.
Bern ranibizumab treatment regimen

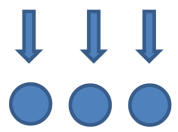

Active disease Loading phase 1st year: Non active

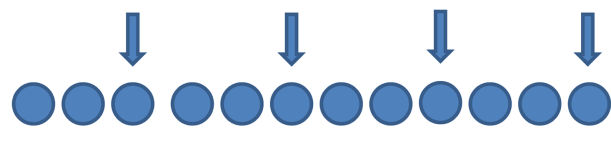

End of therapy
= visit treatment. Three hundred and eightyfive patients were switched to aflibercept during therapy. Therefore, 575 patients received ranibizumab as the only treatment for neovascular AMD within that time period. Out of these patient groups, only 15 patients finished treatment following the complete Bern treatment regime (see figure 2). Only one eye per patient was considered for inclusion since none of the 15 patients had bilateral treatment for exudative AMD. All other patients still needed treatment, or dropped out of the regime for other reasons. Charts of these 15 patients were reviewed, and the following data was analysed: Best corrected ETDRS visual acuity (VA) was tested before the first treatment, at every injection date, and 4 weeks after the last injection. Mean OCT retinal thickness values of the fovea (ETDRS grid) were documented before first treatment, at every injection date, and 4 weeks after the last injection. Structural retinal changes in OCT images were assessed before the first treatment and 4 weeks after the last injection. Retinal morphologic changes, such as subretinal haemorrhages, pigment epithelium detachments, intraretinal and/ or subretinal fluid, geographic atrophy, and fibrovascular scar formation were documented if seen during fundus biomicroscopy or on fundus photographs before treatment and 4 weeks after the last injection. Additionally, fluorescein angiography findings before the first treatment were assessed. Follow-up fluorescein angiography was performed every 6-12 months or at the discretion of the treating physician. Additionally, indocyanin green angiography was performed occasionally if required.

Active CNV was defined as loss of $\geq 5$ letters on the ETDRS chart (not due to scar formation or atrophy), abnormal OCT retinal thickness with signs of intraretinal or subretinal fluid, or new retinal haemorrhage.

Data was analysed using Prism 5 (V.5.02, Graphpad Software, 7825 Fay Avenue, Suite 230, La Jolla, California 92037 USA). Student paired $t$ test was used to compare VA and OCT thickness values before and after treatment. Pairwise correlation was performed using Spearman's coefficient correlation to assess correlation between total number of injections and VA gain, and between total number of injections and change in foveal thickness; $\mathrm{p}<0.05$ were considered statistically significant.

\section{RESULTS}

Fifteen eyes of 15 patients (10 women) with a mean age of 81 \pm 9.9 years (mean \pm SD) were included. No patients had any prior antiVEGF therapy for neovascular AMD. Mean ETDRS VA prior to therapy was $55 \pm 15.4$ letters. Mean ETDRS VA at the end of therapy was $59.6 \pm 17.6$ letters. Mean change in VA was $4.5 \pm 16.9$ letters which was not statistically significant $(p=0.3)$. Mean foveal thickness was significantly thinner after last treatment $(247.9 \pm 43.0 \mu \mathrm{m})$ compared to baseline $(332.5$ $\pm 83.1 \mu \mathrm{m}, \mathrm{p}<0.002)$. Mean change in foveal retinal thickness was $-78.9 \pm 94.1 \mu \mathrm{m}$. The mean total number of ranibizumab injections was $15.6 \pm 8.0$ (min.: 9; max.: 39 injections) and the mean total treatment period was $40.9 \pm 18.3$ months (min.: 27 ; max.: 86 months). VA gain and number of injections were positively correlated $\left(p=0.046, R_{\text {square }}=0.27\right)$. No significant correlation was found for the number of injections and mean foveal thickness $\left(p=0.2, R_{\text {square }}=0.12\right)$ change. Fluorescein angiography prior to treatment revealed 12 cases $(80 \%)$ of occult choroidal neovascularisation (CNV) and 3 cases (20\%) of classic CNV. Table 1 shows additional morphologic characteristics prior to treatment and at the end of therapy.

\section{DISCUSSION}

Although the introduction of antiVEGF therapy for neovascular AMD led to a big improvement in VA compared to the natural course of the disease, there is an ongoing controversy regarding the best treatment regime and tapering treatment. Currently, antiVEGF drugs cannot cure the disease, but at least in the initial stages of therapy, could lead to an improvement in visual acuity. The Bern treatment regime is relatively strict with at least six intravitreal injections of ranibizumab within the first 12 months even if the disease never becomes active again after the loading phase. Even in the optimal course without disease recurrence, patients will receive at least nine injections during the whole treatment regime (see figure 1). The Bern treatment regime offers a precise exit strategy in contrast with many other regimes such as the treat-and-extend, or PRN scheme. To our
Figure 2 Graph indicating the numbers and reasons for inclusion or exclusion of patients.
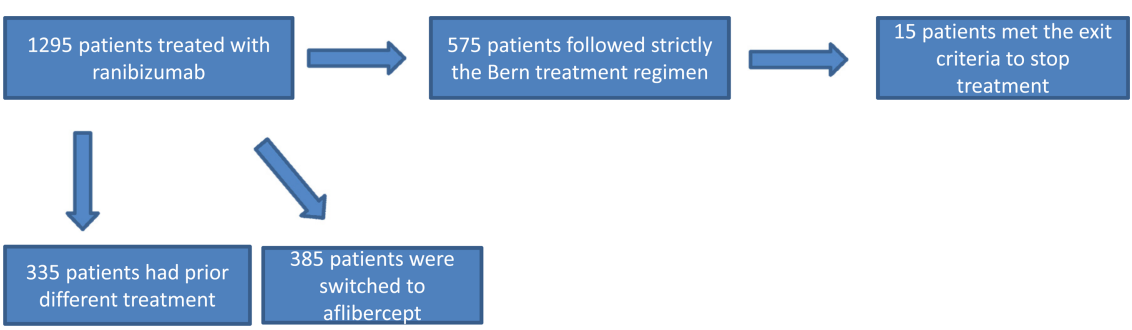
Table 1 Retinal morphologic characteristics prior to treatment and at the end of therapy

\begin{tabular}{lll}
\hline & Pretreatment (\%) & Post-treatment (\%) \\
\hline Geographic atrophy & $3(20)$ & $7(46.6)$ \\
Subretinal fluid & $9(60)$ & 0 \\
Intraretinal fluid & $9(60)$ & $1(0.6)$ \\
pigment epithelium detachment & $2(13.3)$ & $2(13.3)$ \\
Fibrotic scar & 0 & $2(13.3)$ \\
\hline
\end{tabular}

knowledge, this might be the first study reporting the outcome of patients that met the exit criteria of a fixed treatment regimen for exudative AMD. Literature search in pubmed found no relevant data in this matter. However, it was very surprising how few patients actually finished the treatment. Only $2.6 \%$ off all patients in our database finished treatment and reached the exit criteria. Most patients seemed to drop out due to other reasons, or needed continuous treatment. There are many reasons for a patient not to reach the last stages of the maintenance treatment, such as fibrovascular scar formation, very low VA, or decision of the patient not to finish the treatment. Interestingly, 5 out of 15 patients $(33.3 \%)$ only required the minimum nine injections to exit the regime. One can even hypothesise, that the fraction of 15 patients that actually made it to the end of the treatment regime are generally good responders to ranibizumab treatment, compared to all other patients that never met the exit criteria.

Fundus biomicroscopy and OCT revealed subretinal fluid in $60 \%$ of all patients prior to therapy. Additionally, $60 \%$ of patients showed intraretinal fluid at baseline. All patients had either subretinal or intraretinal fluid, or both, at baseline. No patient showed remaining subretinal fluid, and only one patient had some remaining intraretinal fluid at the end of therapy. By contrast, no change was found in the rate of pigment epithelium detachment (13.3\%). Only two eyes (13.3\%) developed new fibrotic scar tissue within the macula area despite antiVEGF therapy. Interestingly, the rate of geographic atrophy increased from three cases $(20 \%)$ prior therapy to seven cases $(46.6 \%)$ after therapy. This increase might be associated with intravitreal ranibizumab treatment. Recently, Grunwald JE et al published work describing risk factors for the development of geographic atrophy in the Comparison of Age-related macular degeneration Treatment Trials (CATT). They analysed 1024 CATT patients ${ }^{14}$ with no geographic atrophy visible at enrolment, and followed patients during 1 year of monthly injections and 1 year of PRN treatment with antiVEGF drugs (ranibizumab or bevacizumab). Approximately one-fifth of CATT patients developed geographic atrophy within 2 years of treatment, and authors concluded that antiVEGF treatment may play a role in the development of geographic atrophy.

In our study group, overall VA gain was $4.5 \pm 16.9$ letters compared to baseline, with a mean follow-up of 40.9 \pm 18.3 months. These findings are comparable with the results of various major studies which reported general stabilisation and/or improvement of VA after intravitreal ranibizumab. ${ }^{4-12}$

Recently, a multicentre cohort study (SEVEN-UP) was published showing the seven-year outcome of eyes treated with ranibizumab in ANCHOR, MARINA and HORIZON. ${ }^{15}$ At a mean of 7.3 years (range, 6.3-8.5 years) after entry into ANCHOR or MARINA, $37 \%$ of study eyes met the primary end point of $20 /$ 70 or better VA, with $23 \%$ achieving a VA of $20 / 40$ or better. Thirty-seven percent of study eyes had VA of 20/200 or worse.
Forty-three percent of study eyes had a stable or improved letter score ( $\geq 0$-letter gain) compared with ANCHOR or MARINA baseline measurements, whereas $34 \%$ declined by 15 letters or more, with an overall mean decline of 8.6 letters $(\mathrm{p}<0.005)$. The study showed that even after 7 years of extensive treatment, neovascular AMD remains a risk for substantial visual loss. Only one-third of patients had good visual outcome, half the patients remained stable, but one-third declined by 15 letters or more despite regular therapy.

Our data underlines the fact that antiVEGF treatment for neovascular AMD is useful and effective in preserving vision in many, but not all patients. There is still no cure for neovascular AMD, and antiVEGF treatment confronts the physician with a number of unsolved problems, such as unknown long-term side effects (ie, geographic atrophy), and lack of alternative treatment options or exit strategies.

There are certain limitations of this study. First, the sample size of patients that met exit criteria is small. Therefore, the outcome of patients might not be representative. The functional and anatomical outcome of all patients who did not meet exit criteria would have been interesting as well, since we do not know why these patients did not respond well enough to treatment. However, that data could not be analysed in this study. Additionally, there is no control group (that would have been treated with another exit strategy) for comparison. Therefore, we do not know if our applied exit strategy is effective and safe in defining end of treatment. Long-term follow-up of these 15 patients would be needed to calculate the recurrence rate after end of therapy over the next couple of years.

In conclusion, our study showed that even with a fixed treatment regime and a defined treatment exit strategy, only a small percentage of patients will actually complete the exit phase.

Contributors MM: Conception, Data analysis, Writing, Final approval MZ: Writing, Final approval AE: Data analysis, Writing, Final approval SW: Critical review, logistical support, final approval.

Competing interests MM and SW have served as consultants and/or speaker for Novartis AG.

Ethics approval IRB approval for this work was obtained.

Provenance and peer review Not commissioned; externally peer reviewed.

Data sharing statement All authors had access to any data upon which the manuscript is based and will provide such data upon request to the editors or their assignees.

Open Access This is an Open Access article distributed in accordance with the Creative Commons Attribution Non Commercial (CC BY-NC 3.0) license, which permits others to distribute, remix, adapt, build upon this work non-commercially, and license their derivative works on different terms, provided the original work is properly cited and the use is non-commercial. See: http://creativecommons.org/ licenses/by-nc/3.0/

\section{REFERENCES}

1 Friedman DS, O'Colmain BJ, Munoz B, et al. Prevalence of age-related macular degeneration in the United States. Arch Ophthalmol 2004;122:564-72.

2 Ferrara N, Gerber HP, LeCouter J. The biology of VEGF and its receptors. Nat Med 2003;9:669-76.

3 AG NP. Product monograph: Lucentis in AMD. 2010.

4 Fung $A E$, Lalwani GA, Rosenfeld PJ, et al. An optical coherence tomography-guided, variable dosing regime with intravitreal ranibizumab (Lucentis) for neovascular age-related macular degeneration. Am J Ophthalmol 2007;143:566-83.

5 Mitchell $P$, Korobelnik JF, Lanzetta $P$, et al. Ranibizumab (Lucentis) in neovascular age-related macular degeneration: evidence from clinical trials. $\mathrm{Br} J$ Ophthalmol 2010:94:2-13.

6 Schmidt-Erfurth UM, Richard G, Augustin A, et al. Guidance for the treatment of neovascular age-related macular degeneration. Acta Ophthalmol Scand 2007:85:486-94.

7 Brown DM, Kaiser PK, Michels $M$, et al. Ranibizumab versus verteporfin for neovascular age-related macular degeneration. N Engl J Med 2006:355:1432-44. 
8 Rosenfeld PJ, Brown DM, Heier JS, et al. Ranibizumab for neovascular age-related macular degeneration. N Engl J Med 2006;355:1419-31.

9 Regillo CD, Brown DM, Abraham P, et al. Randomized, double-masked, sham-controlled trial of ranibizumab for neovascular age-related macular degeneration: PIER Study year 1. Am J Ophthalmol 2008;145:239-48.

10 Boyer DS, Heier JS, Brown DM, et al. A Phase IIlb study to evaluate the safety of ranibizumab in subjects with neovascular age-related macular degeneration. Ophthalmology 2009;116:1731-9.

11 Meyer CHEN, Holz FG. SUSTAIN Study Group: Ranibizumab in patients with subfoveal choroidal neovascularisation secondary to age-related macular degeneration. Interim results from SUSTAIN trial. Invest Ophthalmol Vis Sc 2008;49:273.
12 Bolz MS-EU. Ranibizumab EXCITE study: exploring the value of optical coherence tomography for the management of ranibizumab therapy in age related macular degeneration. Vienna: 8th EURETINA Congress, 2008.

13 Menke MN, Salam A, Framme C, et al. Long-term intraocular pressure changes in patients with neovascular age-related macular degeneration treated with ranibizumab. Ophthalmologica. Ophthalmologica 2013;229:168-72.

14 Martin DF, Maguire MG, Fine SL, et al. Ranibizumab and bevacizumab for treatment of neovascular age-related macular degeneration: two-year results. Ophthalmology 2012;119:1388-98.

15 Rofagha S, Bhisitkul RB, Boyer DS, et al. Seven-Year Outcomes in RanibizumabTreated Patients in ANCHOR, MARINA, and HORIZON: A Multicenter Cohort Study (SEVEN-UP). Ophthalmology 2013;120:2292-9. 